\title{
Non-thermal pulsed X-ray emission from rotation-powered pulsars
}

\author{
L. Zhang ${ }^{1,2}$ and Z. J. Jiang ${ }^{2}$ \\ 1 National Astronomical Observatories/Yunnan Observatory, Chinese Academy of Sciences, PO Box 110, Kunming, China \\ e-mail: astroynu@public.km.yn.cn \\ 2 Department of Physics, Yunnan University, Kunming, China
}

Received 25 January 2005 / Accepted 28 March 2006

ABSTRACT

\begin{abstract}
We study the properties of the pulsed component of hard $(>2 \mathrm{keV}) \mathrm{X}$-ray emission from pulsars based on the new version of the outergap model proposed by Zhang et al. (2004). In this outer-gap model, high-energy photons emitted by relativistic charged particles produce $\mathrm{e}^{ \pm}$pairs through magnetic-pair production on their way from the outer gap to the neutron-star surface, and these pairs produce the bulk of pulsed X-rays by the synchrotron radiation. The X-ray luminosity of rotation-powered pulsars is a function not only of the period and magnetic field strength, but also of the magnetic inclination angle. Application of this model to the observed pulsed X-ray emission of normal pulsars by ASCA shows a better consistence. Further, the inclination angles of these pulsars are estimated using the observed X-ray data, and the predicted conversion efficiencies of high-energy $\gamma$-rays for seven confirmed $\gamma$-ray pulsars are consistent with the observed data.
\end{abstract}

Key words. pulsars: general - radiation mechanisms: non-thermal - X-rays: stars

\section{Introduction}

It is believed that hard $(>2 \mathrm{keV})$ pulsed $\mathrm{X}$-ray emission from a pulsar comes from a pulsar magnetosphere and is dominated by non-thermal radiation. Einstein data indicate a relationship between the X-ray luminosity $L_{\mathrm{x}}$ and spin-down power $L_{\mathrm{sd}}(\mathrm{erg} / \mathrm{s})$ of pulsars as $L_{\mathrm{x}} \propto L_{\text {sd }}^{1.39}$ (Seward \& Wang 1988). Observation of ROSAT and ASCA have given the observed data of more and more rotation-powered pulsars at the X-ray band. Becker \& Trümper (1997) examined the correlation by using 27 spin-down pulsars observed by ROSAT in soft X-ray band $(0.1-2.4 \mathrm{keV})$ and found that $L_{\mathrm{x}} \sim 10^{-3} L_{\mathrm{sd}}$. Furthermore, using the data from ASCA (2-10 keV), Saito (1998) gives the correlation between the X-ray luminosity and the spin-down luminosity and concludes that at the hard X-ray band $L_{\mathrm{x}} \simeq 10^{-21} L_{\mathrm{sd}}^{3 / 2}$. Possenti et al. (2002) have obtained a relation $L_{\mathrm{x}}=10^{-15.3} L_{\mathrm{sd}}^{1.34}$ through a re-examination of 39 pulsars. Recently, Cheng et al. (2004) investigated hard X-ray emission from the non-accreting pulsars using ASCA data, including non-thermal, non-pulsed, and pulsed X-ray components. They collected 23 X-ray pulsars (19 normal X-ray pulsars plus 4 millisecond X-ray pulsars) that have been resolved with both pulsed and non-pulsed components obtained from the ASCA, and found that the best fit between $L_{\mathrm{x}}$ and $L_{\mathrm{sd}}$ for the pulsed components of these X-ray pulsars is $L_{\mathrm{x}, \mathrm{pul}} \simeq(1.0 \pm 0.6) \times 10^{-11} L_{\mathrm{sd}}^{1.20 \pm 0.08}$.

Two kinds of pulsar models can explain the non-thermal $\mathrm{X}$-ray emission from the pulsars, one is polar cap models (e.g. Zhang \& Harding 2000); the other outer gap models (e.g. Halpern \& Ruderman 1993; Zhang \& Cheng 1997; Wang et al. 1998; Cheng et al. 1998; Hirotani \& Shibata 2001). Based on the outer gap model of Zhang \& Cheng (1997), the X-ray properties of rotation-powered pulsars were studied in detail (Cheng et al. 1998; Cheng \& Zhang 1999). Recently, we revised the outer gap model of Zhang \& Cheng (1997) (Zhang et al. 2004); as an approximation, a vacuum solution for the acceleration electric field and a non-vacuum particle density are assumed for explaining the high-energy photon flux.

In this revised model, three important effects have been taken into account. The first is the effect of the inclination angle $(\alpha)$ in determining the size of the outer gap; the second discards the assumption that the typical radiation region of the outer gap is at half of the light cylinder, and an appropriate average over the entire outer gap is used instead. This effect is particularly important for old pulsars. When the gap size at this region is larger than unity, the outer gap is assumed to be turned off; and the third effect is that the outer gap still exists as long as the fractional size of the outer gap at the inner boundary is less than unity. This effect allows some pulsars with the appropriate combination of $\alpha$, $P$, and $B$, to maintain their outer gaps until they are a few million years old. Here, we use a new version of the outer gap model proposed by Zhang et al. (2004) to study the properties of the pulsed $\mathrm{X}$-ray component of the normal pulsars, and compare model results with the observed pulsed X-ray luminosity of the 19 X-ray pulsars collected by Cheng et al. (2004). In Sect. 2 we present the outer-gap model for describing non-thermal X-ray radiation from rotation-powered pulsars. In Sect. 3 we apply the model to 19 known X-ray pulsars, and compare with the observed data by ASCA. Finally we briefly give our discussions and conclusions in Sect. 4.

\section{Non-thermal pulsed X-ray emission in the outer gap model}

In the outer-gap model of Zhang et al. (2004), a criterion for the existence of an outer gap is given in terms of the fractional size of the outer gap, $f$. Inside the outer gap, the curvature photons interact with the thermal X-rays from the stellar surface to produce $\mathrm{e}^{ \pm}$pairs through a photon-photon pair production process, thereby sustaining the outer gap. This pair-production condition is $\left\langle E_{\mathrm{X}}\right\rangle E_{\gamma}\left(1-\cos \left(\theta_{\mathrm{X} \gamma}\right)\right)=2\left(m_{\mathrm{e}} c^{2}\right)^{2}$, where $\left\langle E_{\mathrm{X}}\right\rangle$ is the 
average X-ray energy and $\theta_{\mathrm{X} \gamma}$ the angle between the emission directions of curvature photons and the thermal X-rays. In the self-sustained outer gap model of Zhang et al. (2004), the thermal X-ray come from the bombardment of the relativistic particles from the outer gap. For a given $\gamma$-ray pulsar with period, magnetic field, and inclination angle, $f$ is a function of $P, B$, and radial distance $r$, which is

$f(r, P, B)=\zeta(r, \alpha, P, B) f_{0}(P, B)$

where $f_{0}(P, B) \approx 5.5 P^{26 / 21} B_{12}^{-4 / 7}$ is the fractional size of the outer gap by ignoring the effect of the inclination angle (Zhang \& Cheng 1997). The function $\zeta(r, \alpha, P, B)$ contains the effect of inclination angle, which can be expressed as

$\zeta(r, \alpha, P, B) \approx \zeta_{1} \times \zeta_{2} \times \zeta_{3}$,

where

$\zeta_{1}=\left[\frac{2 \sqrt{1-\frac{3}{4} \frac{r}{R_{\mathrm{L}}} a(\alpha)}}{\sqrt{1-\frac{3}{4} \frac{r}{R_{\mathrm{L}}} a(\alpha)}+\sqrt{1-\frac{r}{R_{\mathrm{L}}} a(\alpha)}}\right]^{4 / 7}$,

$\zeta_{2}=\left[\frac{4}{3} \frac{\left(1-\frac{3}{4} \frac{r}{R_{\mathrm{L}}} a(\alpha)\right)^{3 / 2}}{a^{3 / 2}(\alpha)\left(1-\frac{1}{2} \frac{r}{R_{\mathrm{L}}} a(\alpha)\right) \cos \alpha}\right]^{1 / 7}$

and

$\zeta_{3}=\left(\ln \frac{r}{R}\right)^{\frac{1}{21}}\left(\frac{r}{R_{\mathrm{L}}}\right)^{\frac{13}{14}}\left[1-\frac{3}{4} \frac{r}{R_{\mathrm{L}}} a(\alpha)\right]^{-\frac{3}{14}}$,

and $a(\alpha)=\sin ^{2}\left(\theta_{\mathrm{c}}-\alpha\right) \sin \theta_{\mathrm{c}}$. It should be noted that $f(r, P, B) \sim$ $h / R_{\mathrm{L}}$, where $h$ represents the vertical height of the outer gap, which is controlled by the photon-photon pair production (Cheng et al. 1986, hereafter CHR; Zhang \& Cheng 1997; Cheng et al. 2000).

Obviously, the present model is a modified version of the original outer gap model (CHR), i.e. a vacuum gap. On the assumption of slab-like geometry, CHR solved the Poisson equation and found that the inner boundary is located close to the null charge surface. Therefore, the outer gap in the CHR model extends from the null charge surface to the light cylinder with a constant $f$. The main difference in the gap structure between the CHR model and our model is that the CHR model is a onedimensional gap structure in which the vertical height of the outer gap is constant, while our model is a two-dimensional model in which the gap extends along both the magnetic field lines and trans-field direction; i.e. $f$ is the function of position. Based on physical considerations, Zhang et al. (2004) assumed that $\gamma$-ray luminosity $\sim f^{3}(r, P, B) L_{\mathrm{sd}}$ for any rotation-powered pulsar does not exceed the spin-down power; that is, the fractional size of the outer gap at any position within the gap must not be larger than unity, $f(r, P, B) \leq 1$. From Eq. (1), $f(r, P, B)$ reaches a minimum at the radius $\left(r_{\text {in }}\right)$ of the inner boundary, and then increases with radius for a given pulsar.

For a given pulsar, if $f(r, P, B)<1$ at the inner boundary, the range of the outer gap is from the inner boundary to light cylinder, if $f(r, P, B) \leq 1$ at $r_{\mathrm{c}}$, or to the radius $r_{\mathrm{b}}<r_{\mathrm{c}}$ of some surface if $f(r, P, B)=1$ at $r_{\mathrm{b}}$, where $r_{\mathrm{c}}$ is the radial distance where the last open field line is the tangent to the light cylinder $\left(r_{\mathrm{c}}\right.$ increases with the magnetic inclination angle, $r_{\mathrm{c}}=R_{\mathrm{L}}$ for aligned rotator), and $r_{\mathrm{b}}$ is determined by $f\left(r_{\mathrm{b}}, P, B\right)=1$. Therefore, a selfsustained outer-gap accelerator does not exist if $f\left(r_{\text {in }}, P, B\right)>1$;

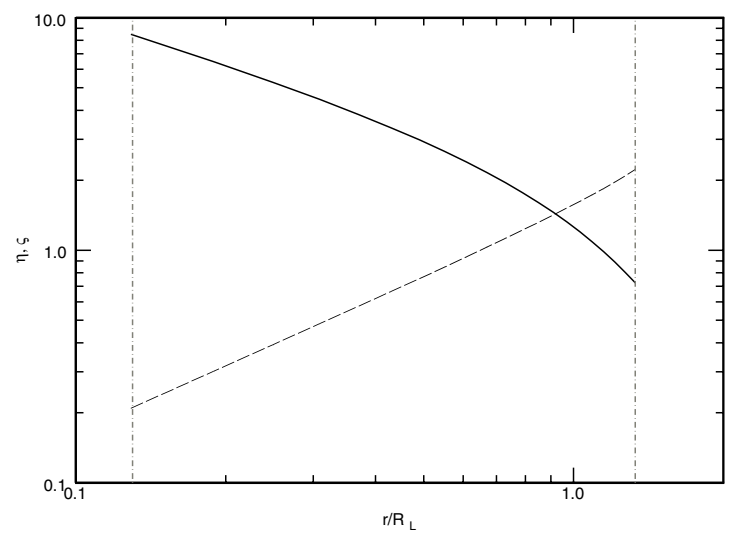

Fig. 1. Variations of $\eta=E_{\|} / E_{0, \|}$ and $\zeta=f(r, P, B) / f_{0}(p, B)$ with radial distance for $\alpha=65^{\circ}$ and Vela pulsar's parameters. Solid and dashed lines represent $\eta$ and $\zeta$, respectively. Tow vertical dotted-dash lines represent the inner and outer boundaries of the outer gap.

otherwise, the outer gap exists and the radius of its outer boundary is $r_{\max }=\min \left(r_{\mathrm{c}}, r_{\mathrm{b}}\right)$. In our model, the parallel electric field, $E_{\|}$, can be estimated when $f$ is given

$E_{\|}=f^{2} B(r)\left(s / R_{\mathrm{L}}\right)=E_{0, \|} \eta(\alpha, r)$,

where

$E_{0, \|}=\frac{64 \pi^{3}}{c^{3}} B_{\mathrm{p}} R^{3} P^{-3} f_{0}^{2}$,

$\eta(\alpha, r)=\zeta^{2}\left(\frac{r}{R_{\mathrm{L}}}\right)^{-5 / 2} \frac{\left[1-(3 / 4) a(\alpha)\left(r / R_{\mathrm{L}}\right)\right]^{2}}{\sqrt{a(\alpha)}\left(1-\frac{1}{2} a(\alpha)\left(r / R_{\mathrm{L}}\right)\right)}$.

Obviously, $E_{\|}$is a function of $\alpha$ and $r$ and decreases with $r$ for a given pulsar. In Fig. 1, we show the variations of $\eta=E_{\|} / E_{0, \|}$ and $\zeta=f(r, P, B) / f_{0}(p, B)$ with a radial distance for $\alpha=65^{\circ}$ and Vela pulsar's parameters; the former decreases with radius, but the latter increases with radius.

It should be pointed out that our model gives an approximate estimation of the outer gap and electric field within the outer gap. Strictly speaking, an electrodynamic study is necessary in order to determine the structure of the outer gap and electric field within the outer gap. Hirotani \& Shibata (1999a,b) propose a one-dimensional non-vacuum outer gap model (also see Hirotani \& Shibata 2001a,b; Hirotani et al. 2003) in which the electrodynamics of the outer gap is studied in detail. In this one-dimensional electrodynamical model, the trans-field thickness (i.e. $f(r, P, B)$ in our model) is assumed to be a constant and is considered as a parameter to be determined by the observed flux (in the sense we cannot compare our model with this onedimensional model).

Because this model neglected important trans-field effects caused by the curvature of the field lines that affect the electrodynamics, Takata et al. (2004) propose a two-dimensional outer gap model with trans-field structure (also see, Takata et al. 2006). After complicated calculations (solving Poisson equation, particle's continuity equations with pair creation model, etc.), they obtained a vacuum and non-vacuum solution with the dipole magnetic field. For the vacuum case, their result indicates the same feature as one of CHR-the inner boundary is located near the null surface-and this feature does not depend on the location of the outer boundary. In this two-dimensional model, one important result for the gap structure is that the gap width relates to trans-field thickness; i.e. large gap width corresponds to a small 
trans-field thickness, at least for the vacuum case, and vice versa (see Figs. 2 and 7 in Takata et al. 2004). Such a feature is similar in our model: a high $f\left(r_{\mathrm{in}}, P, B\right)$ results in a low value for $r_{\mathrm{b}}$ because $f(r, P, B)$ increases with $r$ for a given pulsar, and vice versa. Obviously, there are many differences between our model and the two-dimensional model of Takata et al. (2004). Further detailed study is needed, and is planned for our next paper.

According to Zhang et al. (2004), the Lorentz factor of the primary electrons/positrons at the radius $r$ of the outer gap is

$$
\begin{aligned}
\gamma(r) \approx & 2.84 \times 10^{7} f^{1 / 2}(r) B_{12}^{1 / 4} P^{-1 / 4} \\
& \times\left(1+\frac{3}{4} a(\alpha) \frac{r}{R_{\mathrm{L}}}\right)^{1 / 8}\left(\frac{r}{R_{\mathrm{L}}}\right)^{-3 / 8} .
\end{aligned}
$$

They will lose their energy through curvature radiation, and the characteristic energy of the $\gamma$-ray photons in the outer gap can be approximated as

$$
\begin{aligned}
& E_{\gamma}(r) \approx 143 f^{3 / 2}(r) B_{12}^{3 / 4} P^{-7 / 4}\left(1+\frac{3}{4} a(\alpha) \frac{r}{R_{\mathrm{L}}}\right)^{3 / 8} \\
& \times\left(\frac{r}{R_{\mathrm{L}}}\right)^{-13 / 8} W^{-1 / 4} \mathrm{MeV},
\end{aligned}
$$

where $W(r)=\frac{4}{3}\left[1-\frac{3}{4} a(\alpha) \frac{r}{R_{\mathrm{L}}}\right]^{3 / 2} / \sqrt{a(\alpha)}\left(1-\frac{1}{2} a(\alpha) \frac{r}{R_{\mathrm{L}}}\right)$.

In the outer-gap model, half of the primary electrons/positrons in the outer gap will move toward the star and lose their energy through curvature radiation. The return particle flux can be approximated by $\dot{N}_{\mathrm{e}}=f \dot{N}_{\mathrm{GJ}}$, where $\dot{N}_{\mathrm{GJ}}$ is the Goldreich-Julian current, which is roughly

$\dot{N}_{\mathrm{GJ}} \approx \frac{\Omega^{2} R^{3} B_{\mathrm{p}}}{2 e c} \sin ^{2}\left(\theta_{\mathrm{c}}-\alpha\right) \sin \theta_{\mathrm{c}} \cos \alpha$

in polar coordinates. In such a geometry,

$\boldsymbol{B}(\boldsymbol{r})=\left(B_{\mathrm{p}} R^{3} / 4 r^{3}\right)(2 \cos (\theta-\alpha) \hat{r}+\sin (\theta-\alpha) \hat{\theta})$,

where $B_{\mathrm{p}}$ is estimated by $B_{\mathrm{p}} \approx 6.4 \times 10^{19}(P \dot{P})^{1 / 2} \mathrm{G}, R$ is the stellar radius, and $\hat{r}$ and $\hat{\theta}$ are the unit vectors of radial and polar angle directions, respectively. Halpern \& Ruderman (1993) pointed out that these primary electrons/positrons will radiate most of their energies during the motion from the inner boundary of the outer gap toward the neutron star surface. The inner boundary of the outer gap can be described by $\left(r_{\text {in }}, \theta_{\text {in }}\right)$, which are

$\frac{r_{\text {in }}}{R_{\mathrm{L}}}=\frac{\sin ^{2}\left(\theta_{\text {in }}-\alpha\right)}{\sin \theta_{\mathrm{c}} \sin ^{2}\left(\theta_{\mathrm{c}}-\alpha\right)}$,

and

$\tan \theta_{\text {in }}=\frac{1}{2}\left(3 \tan \alpha+\sqrt{9 \tan ^{2} \alpha+8}\right)$.

According to Zhang \& Cheng (1997), return relativistic particles in the outer gap lose their energy via curvature radiation. We assume that $B(r) \approx 0.5 B_{\mathrm{p}}(R / r)^{3}$ is a reasonable approximation near the surface of the neutron star. In the neutron star's strong magnetic field, these curvature photons will convert into secondary pairs through magnetic-pair production at the distance $r_{\mathrm{s}}$, which is

$r_{\mathrm{s}} \simeq\left(\frac{15 E_{\gamma}\left(r_{\mathrm{in}}\right)}{2 m c^{2}} \frac{B_{\mathrm{p}}}{B_{\mathrm{q}}}\right)^{1 / 3} R$ where $R$ is the neutron-star radius and $B_{\mathrm{q}}=m^{2} c^{3} / e \hbar=4.4 \times$ $10^{13} \mathrm{G}$. These pairs quickly lose their momentum, which is perpendicular to the local magnetic field by synchrotron radiation with the characteristic energy $E_{\mathrm{syn}}=E_{\gamma}\left(r_{\text {in }}\right) / 20$ (Cheng et al. 1998). If the energy of synchrotron photons is high enough, they will convert further into $\mathrm{e}^{ \pm}$in the strong magnetic field until their energy reduces to $E_{\mathrm{f}}$

$E_{\mathrm{f}}=\left(\frac{1}{20}\right)^{n_{\mathrm{f}}} E_{\text {syn }}$

where $n_{\mathrm{f}}$ is the generation number, and this will form a cascade. The position $r_{\mathrm{f}}$ where the pair-production stops is given by (Cheng \& Zhang 1999)

$r_{\mathrm{f}}=\left(\frac{1}{20}\right)^{n_{\mathrm{f}} / 3} r_{\mathrm{s}}$

and then non-thermal X-rays develop. An electromagnetic cascade will take place until the energy of synchrotron photons is $E_{\mathrm{f}}$. According to Zhang \& Cheng (1997), the total number of secondary electron/positron pairs created in the electromagnetic cascade is

$\dot{N} \approx f\left(r_{\text {in }}\right) \dot{N}_{\mathrm{GJ}}\left(\frac{\gamma\left(r_{\text {in }}\right) m c^{2}}{E_{\mathrm{f}}}\right)\left(\frac{r_{\mathrm{s}}}{f\left(r_{\text {in }}\right) r_{\text {in }}}\right)^{2}$.

Therefore the luminosity of non-thermal $X$-rays produced by the cascade is given by $L_{\mathrm{X}}=E_{\mathrm{f}} \times \dot{N}$. Using Eq. (1), we have

$L_{\mathrm{X}}^{n} \simeq 2.8 \times 10^{-6} B_{12}^{0.13} P^{-0.8} \Lambda\left(r_{\mathrm{in}}\right) L_{\mathrm{sd}}$

and

$$
\begin{aligned}
\Lambda\left(r_{\text {in }}\right)= & a(\alpha) \zeta^{1 / 2}\left(r_{\text {in }}\right) \cos \alpha\left[1+\frac{3}{4} a(\alpha) \frac{r_{\text {in }}}{R_{\mathrm{L}}}\right]^{3 / 8} \\
& \times\left(\frac{r_{\text {in }}}{R_{\mathrm{L}}}\right)^{-83 / 24} W^{-1 / 6} .
\end{aligned}
$$

Obviously, for a given pulsar, its X-ray luminosity is a function of the magnetic inclination angle $(\alpha)$.

In order to determine the spectrum of non-thermal X-rays from a pulsar, we need to estimate the minimum $\left(E_{X, \min }\right)$ and maximum $\left(E_{\mathrm{X}, \max }\right)$ energies of these non-thermal X-rays, which are

$E_{\mathrm{X}, \min } \simeq 5 B_{12}\left(r_{\mathrm{f}} / R\right)^{-3} \mathrm{keV}$

and

$E_{\mathrm{X}, \max }=\left(\frac{1}{20}\right)^{n_{\mathrm{f}}+1} E_{\gamma}\left(r_{\mathrm{in}}\right)$

respectively, where $n_{\mathrm{f}}$ is the generation number. Assuming $\beta_{\mathrm{f}}$ is the spectral index at position $r_{\mathrm{f}}, \beta_{\mathrm{f}}=2$ when the cascade approaches infinity, so we assume that $\beta_{\mathrm{f}}$ is generally less than 2 . The spectrum of non-thermal X-rays with a spectral index of $\beta_{\mathrm{f}}$ distributes between $E_{\mathrm{X} \text {,min }}$ and $E_{\mathrm{X} \text {,max }}$, and the differential luminosity of these non-thermal X-rays from the X-ray pulsar is given by

$L_{\mathrm{X}}^{n}\left(E_{\mathrm{X}}\right) \approx \frac{\left(2-\beta_{\mathrm{f}}\right) L_{\mathrm{X}}^{n}}{1-\left(E_{\mathrm{X}, \min } / E_{\mathrm{X}, \max }\right)^{-\beta+2}} \times\left(\frac{E_{\mathrm{X}}}{E_{\mathrm{X}, \max }}\right)^{-\beta_{\mathrm{f}}+1}$, 
Table 1. Pulsed X-ray properties of rotation-powered pulsars. Comparison of model X-ray luminosity $\left(L_{\mathrm{X}}^{\mathrm{th}}\right)$ (assuming $\alpha=55^{\circ}$ ) with observed pulsed X-ray luminosity $\left(L_{\mathrm{X}}^{\mathrm{ob}}\right)$ for each X-ray pulsars.

\begin{tabular}{llllll}
\hline \hline PSR & $P$ & $B_{12}$ & $d$ & $\begin{array}{l}\log \left(L_{\mathrm{X}}^{\mathrm{th}}\right) \\
\mathrm{erg} / \mathrm{s}\end{array}$ & $\begin{array}{l}\log \left(L_{\mathrm{X}}^{\mathrm{ob}}\right) \\
\mathrm{erg} / \mathrm{s}\end{array}$ \\
\hline $\mathrm{J} 0631+1036$ & 0.288 & 10.86 & 1.0 & 30.78 & 31.30 \\
$\mathrm{~J} 1811-1926$ & 0.065 & 3.42 & 5.0 & 32.81 & 33.28 \\
$\mathrm{~B} 0531+21$ & 0.033 & 7.53 & 2.0 & 34.98 & 35.83 \\
$\mathrm{~B} 0833-45$ & 0.089 & 6.75 & 0.3 & 32.79 & 32.18 \\
B0633+17 & 0.237 & 3.28 & 0.16 & 30.06 & 29.66 \\
$\mathrm{~B} 1706-44$ & 0.1025 & 6.25 & 1.82 & 32.42 & 32.61 \\
$\mathrm{~B} 1509-58$ & 0.150 & 30.76 & 4.3 & 33.12 & 34.11 \\
$\mathrm{~B} 1951+32$ & 0.0395 & 0.97 & 2.5 & 32.68 & 32.79 \\
$\mathrm{~B} 1046-58$ & 0.124 & 6.98 & 2.98 & 32.13 & 32.74 \\
$\mathrm{~B} 1929+10$ & 0.227 & 1.04 & 0.17 & 29.07 & 29.74 \\
$\mathrm{~B} 0656+14$ & 0.385 & 9.31 & 0.76 & 30.02 & 31.00 \\
B0540-69 & 0.05 & 9.91 & 49.4 & 34.36 & 36.11 \\
B0950+08 & 0.253 & 0.49 & 0.12 & 28.14 & 29.20 \\
$\mathrm{~B} 1610-50$ & 0.232 & 21.64 & 7.26 & 31.88 & 33.48 \\
B1055-52 & 0.197 & 2.17 & 1.53 & 30.06 & 30.30 \\
$\mathrm{~B} 1853+01$ & 0.267 & 20.43 & 2.02 & 31.53 & 32.15 \\
$\mathrm{~J} 2229+6114$ & 0.0516 & 4.06 & 3.0 & 33.46 & 32.60 \\
B0537-69 & 0.016 & 1.83 & 47.0 & 35.17 & 35.23 \\
$\mathrm{~J} 1846-0258$ & 0.32 & 96.47 & 19.0 & 32.61 & 34.60 \\
\hline
\end{tabular}

Note: the pulsed X-ray luminosity of pulsars have $30 \%$ uncertainty except for B1706-44, B1046-58, and J1610-50 which have a 50\% uncertainty (Cheng et al. 2004). The magnetic field strength is given by $B_{12} \approx 6.4 \times 10^{7}(P \dot{P})^{1 / 2}$.

where $E_{\mathrm{X} \text {,min }} \leq E_{\mathrm{X}} \leq E_{\mathrm{X} \text {,max }}$. For the luminosity of non-thermal $\mathrm{X}$-rays with energy ranges from $E_{1}$ to $E_{2}$, we have

$$
\begin{aligned}
L_{\mathrm{X}}^{n}\left(E_{1}<E<E_{2}\right)= & \left(\frac{E_{2}}{E_{\mathrm{X}, \max }}\right)^{2-\beta_{\mathrm{f}}} \\
& \times \frac{1-\left(E_{1} / E_{2}\right)^{2-\beta_{\mathrm{f}}}}{1-\left(E_{\mathrm{X}, \min } / E_{\mathrm{X}, \max }\right)^{2-\beta_{\mathrm{f}}}} L_{\mathrm{X}}^{n} .
\end{aligned}
$$

\section{Applications}

Cheng et al. (2004) have collected the pulsed X-ray data of 23 X-ray pulsars by ASCA, including 19 normal pulsars and 4 millisecond pulsars (see their Table 1). Here we only consider the hard-pulsed X-ray emission of 19 normal pulsars. The best fit of the observed pulsed X-ray luminosity of the 19 pulsars is $\log L_{\mathrm{X}}=-(9.9 \pm 3.74)+(1.17 \pm 0.10) \log L_{\mathrm{sd}}$. Because the fractional size of the outer gap is the function of magnetic inclination angle $\alpha$ but $\alpha$ is not known well (see Zhang et al. 2004 in detail), we use the inclination angle as a parameter. In Table 1, the typical parameters and expected values for 19 pulsars with $\alpha=55^{\circ}$ are given.

In estimating the X-ray luminosity in the range of $2-10 \mathrm{keV}$ using this model, at first, the fractional size of the outer gap, the typical energy of $\gamma$-rays at the inner boundary of the outer gap and the distance $r_{\mathrm{s}}$ have been calculated for each X-ray pulsar, then the spectral index for the X-ray spectrum and the minimum and maximum X-ray energies of each X-ray pulsar are determined. For each X-ray pulsar, the generation number and spectral index $\beta_{\mathrm{f}} \simeq 1.75$ can be determined based on the $\gamma$-ray energy at the inner boundary. In Fig. 1, the data points from Cheng et al. (2004) are reproduced with the corresponding model values, pulsars with detected $\gamma$-ray emission and the pulsars with

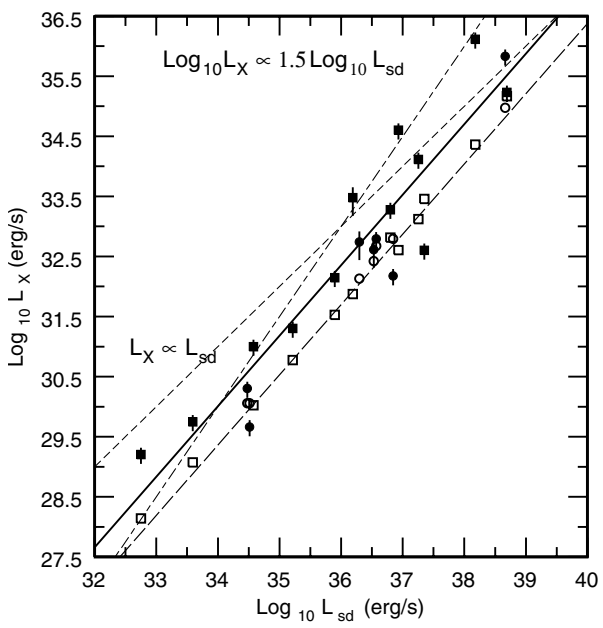

Fig. 2. Plot of the X-ray pulsar luminosity vs. the spin-down luminosity for 19 pulsars. The data points are represented by filled symbols (after Cheng et al. 2004), while model values are represented by open symbols. Pulsars with detected $\gamma$-ray emission are marked by circles and those with no $\gamma$-rays detected are marked by squares.

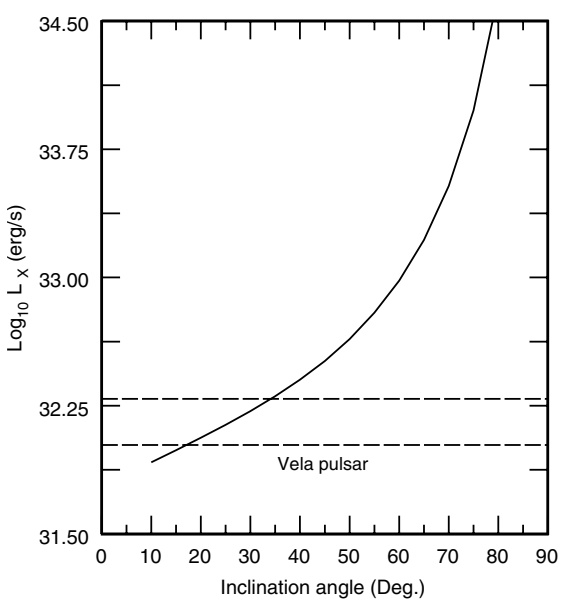

Fig. 3. X-ray luminosity of the Vela pulsar versus the inclination angle. Two horizonal dashed lines represent the upper and lower limits of the observed pulsed X-ray luminosity of the Vela pulsar.

no detected $\gamma$-ray emission. The model results with $\alpha=55^{\circ}$ for 19 pulsars are also shown in Fig. 2, and the best fit is

$L_{\mathrm{X}}=10^{-10.29 \pm 0.70} L_{\mathrm{sd}}^{1.17 \pm 0.02}$,

which is consistent with the observed data. From Fig. 2, the relationship between $L_{\mathrm{X}}(2-10 \mathrm{keV})$ and $L_{\mathrm{sd}}$ in this model satisfies $L_{\mathrm{X}} \propto L_{\mathrm{sd}}^{1.17}$, which is similar to $L_{\mathrm{X}} \simeq 10^{-3} L_{\mathrm{sd}}$ (Becker \& Trümper 1997) and $L_{\mathrm{X}} \simeq 10^{-15.3} L_{\text {sd }}^{1.34}$ (Possenti et al. 2002), but is different from $L_{\mathrm{X}} \simeq 10^{-21} L_{\text {sd }}^{1.5}$ (Saito 1998).

It can be seen from Fig. 2, as pointed out by Cheng et al. (1998), that discrepancies between the observed and the model values of $L_{X}$ result from the actual value of the inclination angle. Therefore, the actual distribution of inclination angles is probably broader, which would automatically imply wider scatter in the X-ray luminosity. In fact, we can show the change in $L_{\mathrm{X}}$ with the inclination angle for each pulsar. For example, we show the change of $L_{\mathrm{X}}$ with $\alpha$ of Vela and Geminga pulsars in Figs. 3 and 4, respectively. It can be seen that the non-thermal X-ray luminosity increases with the inclination angle. Such a feature makes it possible to estimate the inclination angle for each pulsar using the observed data. We fit the 


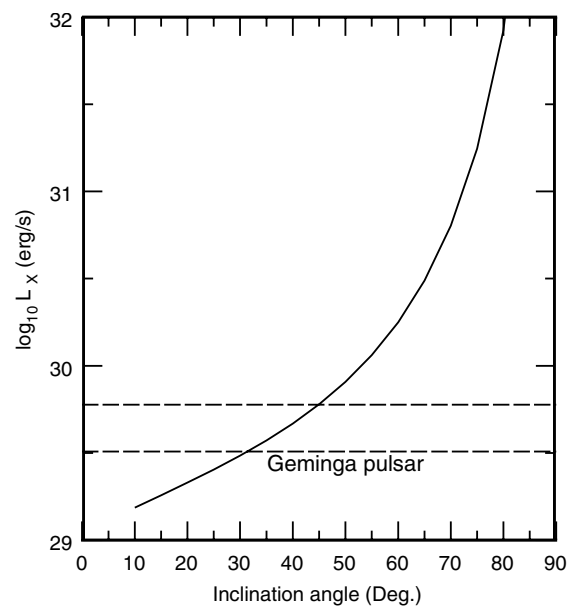

Fig. 4. X-ray luminosity of the Geminga pulsar versus the inclination angle. Two horizonal dashed lines represent the upper and lower limits of the observed pulsed X-ray luminosity of the Geminga pulsar.

observed data of 19 X-ray pulsars by changing the inclination angle for each pulsar. Following Possenti et al. (2002), we grouped these pulsars into four classes: Crab-like, Velalike, Geminga-like, and old pulsars. For the Crab-like pulsars (Crab, PSR B1509-58, PSR B0540-69, PSR B1853+01, PSR B0537-69, and PSR J1846-0258), the inclination angles are large $\left(\geq 70^{\circ}\right)$, the main reason being not knowing the non-thermal X-rays produced in the outer gap through synchrotron and inverse-Compton scattering (Cheng et al. 2000). For the Vela-like pulsars (Vela, PSR J0631+1036, PSR J18111925, PSR B1706-44, PSR B1046-58, PSR B1953+01, and PSR J1846-0258), the inclination angles change from $11^{\circ}$ for PSR J2229+6114 to $\sim 68^{\circ}$ for PSR B1046-58, which indicates a trend of the inclination angle to increase with the pulsar's age. For Geminga-like pulsars (Geminga, PSR B1055-52, PSR B1951+32, and PSR B0656+14), the ranges of the inclination angle are from $40^{\circ}$ for the Geminga pulsar to $\sim 73^{\circ}$ for PSR B0656+14; and we noted that the distances of PSR B0656+14 and PSR B1055-52 used by Cheng et al. (2004) are $0.76 \mathrm{kpc}$ and $1.53 \mathrm{kpc}$, respectively (also see Table 1), which are about twice the present distances $(0.3 \mathrm{kpc}$ and $0.75 \mathrm{kpc})$ of these two pulsars (De Luca et al. 2004). When the new distances are used, the inclination angles for PSR B1055-52 and PSR B0656+14 are $\sim 40^{\circ}$ and $\sim 59^{\circ}$, respectively. The inclination angles for two old pulsars of PSR B1929+10 and PSR B0950+08 are estimated as $\sim 69^{\circ}$ and $\sim 74^{\circ}$, respectively.

Once the inclination angle of a pulsar is given, we can estimate its high-energy $\gamma$-ray emission. Seven pulsars have been confirmed as emitting high-energy ( $\geq 100 \mathrm{MeV}) \gamma$-rays (Hartman et al. 1999; Kaspi et al. 2000), and 19 plausible associations between the unidentified EGRET sources and the observed radio pulsars have been found (Camilo et al. 2000; Torres et al. 2001; Kramer et al. 2003). To explain the average properties of high-energy photon emission from the outer gap, Zhang et al. (2004) assumed that high-energy emission at a average radius $\bar{r}$ represents the typical emission of high-energy photons from a pulsar, the average radius is

$\bar{r}=\frac{\int_{r_{\text {in }}}^{r_{\max }} f(r, \alpha) r \mathrm{~d} r}{\int_{r_{\text {in }}}^{r_{\max }} f(r, \alpha) \mathrm{d} r}$.

The radius $\bar{r}$ for a pulsar with a given inclination angle $(\alpha)$ can be determined, which is a function of $P, B$, and $\alpha$. Therefore the typical $\gamma$-ray luminosity of pulsars is $L_{\gamma}=f^{3}(\bar{r}, P, B) L_{\mathrm{sd}}$, where $L_{\mathrm{sd}}$ is the pulsar spin-down power. When the pulsar's inclination angles are given, we can estimate the conversion efficiency from rotation power to $\gamma$-ray power for the pulsar $\left(\eta_{\gamma}^{\text {th }}\right)$, which is defined as $\eta_{\gamma}^{\text {th }}=L \gamma / L_{\mathrm{sd}}$. Using our estimate of the inclination angles, we calculated the conversion efficiencies of seven confirmed $\gamma$-ray pulsars and compared them with the observed data $\left(\eta_{\gamma}^{\mathrm{ob}}\right)$, where a solid angle of $\gamma$-ray beam is assumed to be 1 sr when $\eta_{\gamma}^{\text {ob }}$ is determined (Fierro 1995). We show our results in Table 2, where it can be seen that the model results are consistent with the observed data.

\section{Conclusion and discussion}

Based on a new version of the outer-gap model proposed by Zhang et al. (2004), we study the pulsed non-thermal X-ray emission from normal pulsars. In this model, the effects of magnetosphere geometry and inclination angle are taken into account, so the fractional size of the outer gap is a function of period, magnetic field strength, inclination angle, and the distance to the neutron star.

In our model, both outward and inward gamma-rays are produced in the outer gaps, but inward and/or outward $\mathrm{X}$-rays depend on the pair- production processes in the outermagnetosphere. For most pulsars except for the crab-like pulsars, the optical depth due to photon-photon pair production at the typical distance of the outer gap is small, so only a small portion of the outward gamma-rays materializes due to photonphoton pair production and most high-energy outward gammarays can escape from the outer gap (see Zhang \& Cheng 2002). Because most of the inward gamma-rays materialize due to magnetic pair production for the normal pulsars, in particular for large inclination angles, resulting in inward X-ray luminosity is three order of magnitude larger than the outward ones for the mature pulsars (Cheng et al. 2000), gamma-ray emission are dominated by the outward gamma-rays but non-thermal X-ray emission are dominated by inward X-rays except for the crablike pulsars.

We applied this model to 19 normal X-ray pulsars whose pulsed X-ray components in the energy band of $2-10 \mathrm{keV}$ have been detected by ASCA (see Table 1). For simplicity, we assumed that $\alpha=55^{\circ}$ in our calculations although different pulsars may have different inclination angles. The predicted X-ray luminosity for these pulsars are shown in Fig. 2, where they are shown to be consistent with the observed data. Further, our model indicates that X-ray luminosity for each pulsar increases with the inclination angle of this pulsar (see Figs. 3 and 4), so using X-ray data of these pulsars and this feature of our model, we estimated the approximate inclination angles of these pulsars, and then calculated high-energy $\gamma$-ray conversion efficiencies for the confirmed $\gamma$-ray pulsars, which are consistent with the observed data (see Table 2).

The differences between the model of Cheng et al. (1998) (also see Cheng \& Zhang 1999) and this model are (i) Cheng et al. (1998) use the average fractional size of the outer gap, which does not depend on the inclination angle (i.e. $f=f_{0}$ ), but the fractional size of the outer gap in this model is a function of the inclination angle, i.e. $f=f_{0} \zeta(\alpha)$; and (ii) they assume that the cascade stops when the final energy of synchrotron radiation is $E_{0} \sim 1 \mathrm{MeV}$, but here is $E_{0}=E_{\mathrm{f}}$. Although there are these differences, both models have similar results. The former gives $L_{\mathrm{X}} \propto L_{\mathrm{sd}}^{1.15}$ for the pulsed X-ray luminosity of 14 pulsars (Cheng \& Zhang 1999), the latter gives $L_{\mathrm{X}} \propto L_{\mathrm{sd}}^{1.17}$ 
Table 2. Comparison of observed and theoretical $\gamma$-ray luminosity for confirmed high-energy $\gamma$-ray pulsars.

\begin{tabular}{cccccccc}
\hline \hline PSR & $\begin{array}{c}P \\
(\mathrm{~s})\end{array}$ & $\begin{array}{c}B_{12} \\
(\mathrm{G})\end{array}$ & $\begin{array}{c}d \\
(\mathrm{kpc})\end{array}$ & $\begin{array}{c}\alpha \\
(\mathrm{deg} .)\end{array}$ & $f(\bar{r})$ & $\begin{array}{c}\eta_{\gamma}^{\text {th }} \\
(\%)\end{array}$ & $\begin{array}{c}\eta_{\gamma}^{\text {ob }} \\
(\%)\end{array}$ \\
\hline B0531+21 & 0.033 & 7.53 & 2.0 & $\sim 72$ & 0.04 & 0.006 & $0.013 \pm 0.006$ \\
B0833-45 & 0.089 & 6.75 & 0.3 & $\sim 28$ & 0.10 & 0.102 & $0.065 \pm 0.025$ \\
B0633+17 & 0.237 & 3.28 & 0.16 & $\sim 40$ & 0.53 & 15.0 & $5.0 \pm 4.0$ \\
B1706-44 & 0.1025 & 6.25 & 1.82 & $\sim 60$ & 0.15 & 0.35 & $0.72 \pm 0.47$ \\
B1951+32 & 0.0395 & 0.97 & 2.5 & $\sim 58$ & 0.13 & 0.23 & $0.26 \pm 0.17$ \\
B1046-58 & 0.124 & 6.98 & 2.98 & $\sim 68$ & 0.20 & 0.82 & $1.1 \pm 0.3$ \\
B1055-52 & 0.197 & 2.17 & 1.53 & $\sim 63$ & 0.64 & 25.6 & $15 \pm 7$ \\
\hline
\end{tabular}

Note: the inclination angle for each pulsar is estimated by using X-ray observed data in the frame of our model.

for the pulsed X-ray luminosity of 19 pulsars, indicating that the previous model for X-ray emission from pulsars (for example Cheng \& Zhang 1999) is reasonable.

From Fig. 2, there is a certain difference between the different observed data with model results for each pulsar. According to this model, the difference could possibly come from (i) the actual value of the inclination angle and viewing angle and/or from (ii) ignoring the contribution of the non-thermal X-rays indirectly emitted from the outer gap for the Crab-like pulsars (Cheng et al. 2000; Zhang \& Cheng 2000). It should be pointed out that our estimate of pulsar inclination angle depends on the pulsar distance, but some pulsar's distances are not welldetermined, which introduces a bigger uncertainty for the estimate of the inclination angle.

Acknowledgements. We thank the anonymous referee for his very constructive comments and K.S. Cheng for his helpful suggestions. This work is partially supported by "Hundred Talents Program of CAS", Grant for Distinguished Young Scientists from NSFC (10425314), Grand from NSFC (10463002), and Grant from Yunnan Province (2004PY01).

\section{References}

Becker, W., \& Trümper, J. 1997, A\&A, 326, 682

Camilo, F., Bell, J. F., Manchester, R. N., et al. 2001, ApJ, 557, L51
Cheng, K. S., \& Zhang, L. 1999, ApJ, 515, 337

Cheng, K. S., Ruderman, M., \& Zhang, L. 2000, ApJ, 537, 964

Cheng, K. S., Gil, J., \& Zhang, L. 1998, ApJ, 493, L35

Cheng, K. S., Taam, R. E., \& Wang, W. 2006, ApJ, 641, 427

De Luca, A., Caraveo, P. A., Mereghetti, S., et al. 2005, ApJ, 623, 1051

Fierro, J. M. 1995, Ph.D. Thesis, Stanford Univ.

Halpern, J. P., \& Ruderman, M. A. 1993, ApJ, 415, 286

Hartman, R. C., Bertsch, D. L., Bloom, S. D., et al. 1999, ApJS, 123, 79

Hirotani, K., \& Shibata, S. 1999a, MNRAS, 308, 54

Hirotani, K., \& Shibata, S. 1999b, MNRAS, 308, 67

Hirotani, K., \& Shibata, S. 2001a, MNRAS, 325, 1228

Hirotani, K., \& Shibata, S. 2001b, ApJ, 558, 216

Hirotani, K., Harding, A. K., \& Shibata, S. 2003, ApJ, 591, 334

Kaspi, V. M., Lackey, J. R., Mattox, J., et al. 2000, ApJ, 528, 445

Kramer, M., Bell, J. F., Manchester, R. N., et al. 2003, MNRAS, 342, 1299

Possenti, A., Cerutti, R., Colpi, M., et al. 2002, A\&A, 387, 993

Saito, Y. 1998, Ph.D. Thesis, Univ. of Tokyo

Seward, F. D., \& Wang, Z. 1988, ApJ, 332, 199

Takata, J., shibata, S., \& Hirotani, K. 2004, MNRAS, 354, 1120

Takata, J., shibata, S., Hirotani, K., \& Chang, H.-K. 2006, MNRAS, 366, 1310

Torres, D. F., Butt, Y. M., \& Camilo, F. 2001, ApJ, 560, L155

Wang, F. Y.-H., Ruderman, M., Halpern, J. P., \& Zhu, T. 1998, ApJ, 498, 373

Zhang, B., \& Harding, A. K. 2000, ApJ, 532, 1150

Zhang, L., \& Cheng, K. S. 1997, ApJ, 487, 370

Zhang, L., \& Cheng, K. S. 2000, A\&A, 363, 575

Zhang, L., \& Cheng, K. S. 2002, ApJ, 579, 716

Zhang, L., Cheng, K. S., Jiang, Z. J., \& Leung, P. 2004, ApJ, 604, 317 